International Journal of Engineering and Technology, 1 (4) (2012) 483-504

(C) Science Publishing Corporation

www.sciencepubco.com/index.php/IJET

\title{
DWT-based Illumination Normalization and Feature Extraction for Enhanced Face Recognition
}

\author{
K Manikantan ${ }^{1}$, Milan S Shet ${ }^{1}$, Minal Patel $^{1}$, S Ramachandran ${ }^{2}$ \\ ${ }^{1}$ Department of Electronics and Communication Engineering, \\ M S Ramaiah Institute of Technology, Bangalore, Karnataka, India 560054 \\ E-mail: kmanikantan@msrit.edu, milanshet@gmail.com,minal.patel148@gmail.com \\ ${ }^{2}$ Department of Electronics and Communication Engineering, \\ S J B Institute of Technology, Bangalore, Karnataka, India 560060 \\ E-mail: ramachandr@gmail.com
}

\begin{abstract}
Face recognition (FR) under varying lighting conditions is challenging, and exacting illumination invariant features is an effective approach to solve this problem. In this paper, we propose to utilize Discrete Wavelet Transform (DWT) for normalizing the illumination variance in images as well as for feature extraction. Individual stages of the FR system are examined and an attempt is made to improve each stage. A Binary Particle Swarm Optimization (BPSO) based feature selection algorithm is used to search the feature space for the optimal feature subset. Experimental results, obtained by applying the proposed algorithm on YaleB and Color FERET face databases, show that the proposed system outperforms other FR systems. A significant increase in the recognition rate and a substantial reduction in the number of features is observed. Dimensionality reduction obtained is more than $99 \%$ for both YaleB and Color FERET databases.
\end{abstract}

Keywords: Discrete Wavelet Transform, Face Recognition, Feature Extraction, Illumination Normalization, Particle Swarm Optimization.

\section{Introduction}

Face recognition technologies [1, 2, 3, 4] have been widely applied in the areas of intelligent surveillance, identity authentication, human-computer interaction, 
and digital amusement. However, one of the main limitations in deploying face recognition systems for practical use is their relatively low performance due to illumination variations [5, 6, 7, 8]. So face illumination normalization is a central task in face recognition, and many algorithms have been developed to tackle this issue.

Training and recognition form the major stages in FR process. Training stage involves pre-processing, feature extraction, feature selection and creation of a face gallery [9, 10, 11]. Recognition stage involves pre-processing of the test images, feature extraction, feature selection and classification/identification of these images.

For databases with illumination variance such as YaleB and Color FERET, edge detection is necessary for feature extraction. To improve the recognition rate of the FR system on images with varying illumination conditions, we propose a DWT based pre-processing technique named as 2D-DWT based Multilevel Illumination Normalization (DWT-MIN). This technique normalizes the illumination variant images by decomposing the image in the frequency domain. The high frequency and low frequency components are manipulated separately to obtain a normalized image. The required facial features are then extracted from the pre-processed image using again the DWT from a Haar Wavelet Transform. Feature selection process is accomplished using Binary Particle Swarm Optimization (BPSO).

The rest of the paper is organized as follows: Section 2 deals with the fundamental pre-processing methodologies. Section 3 introduces the proposed DWT-based Multilevel Illumination Normalization (DWT-MIN). Section 4 presents the DWT technique for feature extraction. Feature selection using BPSO is dealt with in Section 5. Euclidean distance as a metric for comparison is explained in Section 6. The experimental results and discussions are presented in Section 7 and finally the conclusions in Section 8 .

\section{Basic Image Pre-processing}

The block diagram of the proposed FR system is shown in Fig. 1. It shows the various steps involved in processing an input image. Fig. 2 shows the various pre-processing steps.

Resolution Reduction using Bi-cubic Interpolation reduces the dimensionality of the data which speeds up the computations, without losing too much information. Bi-cubic interpolation, in which the output pixel values are calculated from a weighted average of pixels in the nearest 4-by-4 neighborhood [12], produces noticeably sharper images than any other non-adaptive interpolation algorithms, and is perhaps the ideal combination of processing time and output quality. In our experiments, 50\% resolution reduction has been done for all YaleB and Color FERET images before applying DWT-MIN. 
Gamma Intensity Correction (GIC) is an image enhancement technique and is employed to images with varying illumination conditions to neutralize the impact of illumination [4, 12]. Gamma correction, also known as power law transformation, has its basic form as

$$
O(u, v)=c * I(x, y)^{\gamma}
$$

where $\mathrm{O}(\mathrm{u}, \mathrm{v})$ - gamma corrected image, c - a constant, $\mathrm{I}(\mathrm{x}, \mathrm{y})$ - input image, and $\gamma$ - correction factor.

An edge is a set of pixels in which the image intensity rapidly changes. The edge detection process serves to simplify the analysis of images by drastically reducing the amount of data to be processed, while at the same time preserving useful structural information about object boundaries [13. The Laplacian of Gaussian (LoG) edge detector finds edges by looking for zero crossings after filtering the image with a LoG filter [14].

The behavior of the four pre-processing steps on a sample illumination

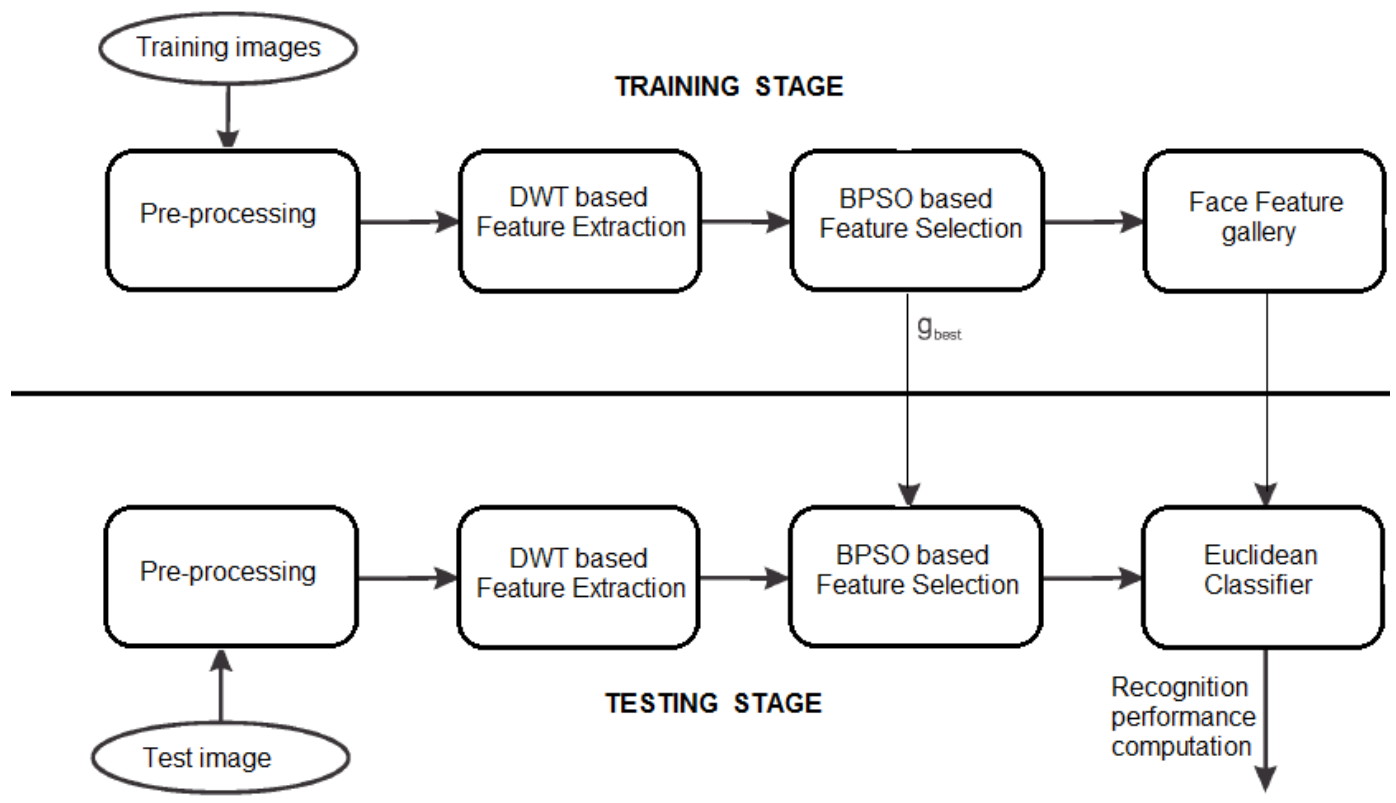

Figure 1: Block Diagram of the proposed FR system.

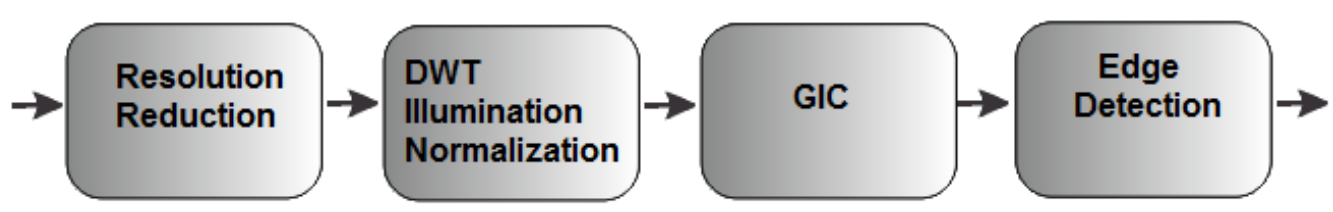

Figure 2: Pre-processing steps. 


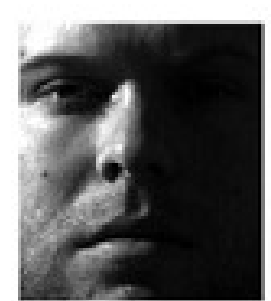

(a)

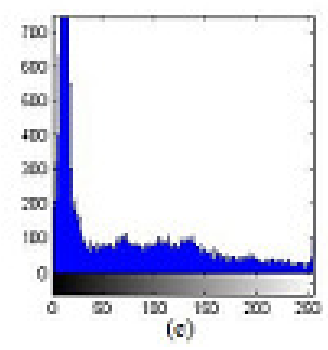

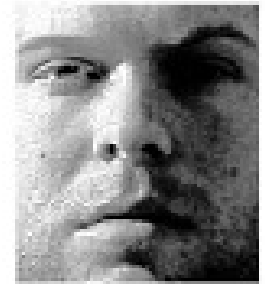

(b)

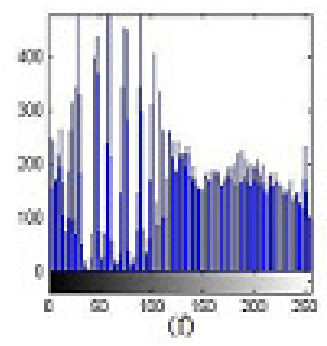

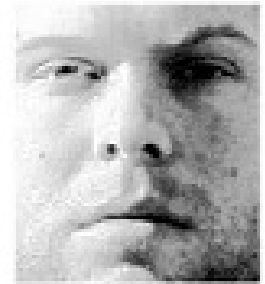

(c)

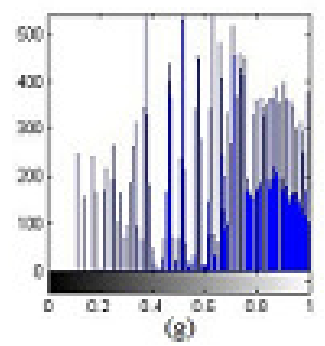

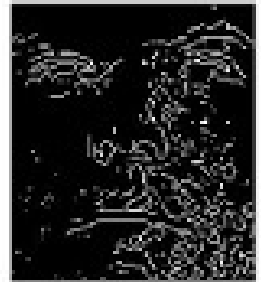

(d)

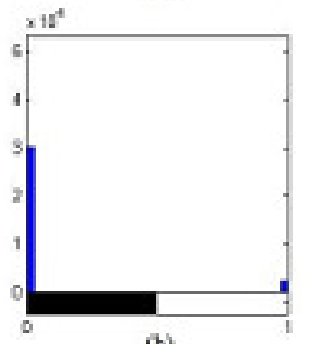

(h)

Figure 3: (a) Sample Image from YaleB Database. (b) Image after applying the proposed DWT-MIN. (c) Image after Gamma Intensity Correction (normalized xaxis). (d) Image after Edge detection. (e) - (h) Corresponding histograms.

variant image from the YaleB database is shown in Fig. 3 .

\section{Proposed DWT based Multilevel Illumina- tion Normalization (DWT-MIN)}

Extrinsic factors like varying illumination conditions could pose a problem in FR. These illumination problems can be solved using illumination normalization. Wavelet based illumination normalization of the face image is a photometric normalization technique done using the 2D - Multilevel Haar Wavelet Transform [8, 15]. The proposed DWT-MIN uses the 2D - Multilevel Haar Wavelet Transform to decompose an image into approximation coefficients and detail coefficients at different levels. Fig. 4 shows the decomposition process by applying the 2D-DWT on a face image. The original image in Fig. 4(a) is decomposed into four sub-band images in Fig. 4(b). Similarly, 2 levels of the Wavelet decomposition as shown Fig. 4(c) can be obtained by applying the wavelet transform on the low frequency band sequentially. At a certain decomposition level, the approximation coefficient matrix depicts the insensitive features, and the three detail coefficient matrices depict the pose, expression and structure features [11] of the approximation coefficient matrix of the previous decomposition level. In this technique, contrast and edge enhancements are done at each level. Contrast enhancement is achieved by histogram equal- 
ization of the approximation coefficients. Histogram equalization modifies the dynamic range (contrast range) of the image and as a result, some important facial features become more apparent [16]. Edge enhancement is done to emphasize the fine details in the original image. The perceptibility of edges and small features can be improved by enlarging the amplitude of the high frequency components in the image. To accentuate details, we multiply each element in the detail coefficient matrices with a scale factor Sf (> 1) [5]. As the decomposition level increases, the contrast and edges are enhanced further. A normalized image is obtained from the modified coefficients by inverse DWT. This normalized reconstructed image is given to the next level for further contrast and edge enhancements. It is to be noted that this process does not alter the resolution of the image. The flowchart for the proposed Multilevel Illumination Normalization is shown in Fig. 5.

Assume $\mathrm{N}$ to be the required wavelet decomposition level. The algorithm for the proposed DWT-MIN is:

$$
\text { for }(\mathrm{i}=1: \mathrm{N})
$$

1. Adjust the dynamic range of the gray scale image to the interval $[0,255]$.

2. Apply single level DWT to the image and obtain the approximate, ver-

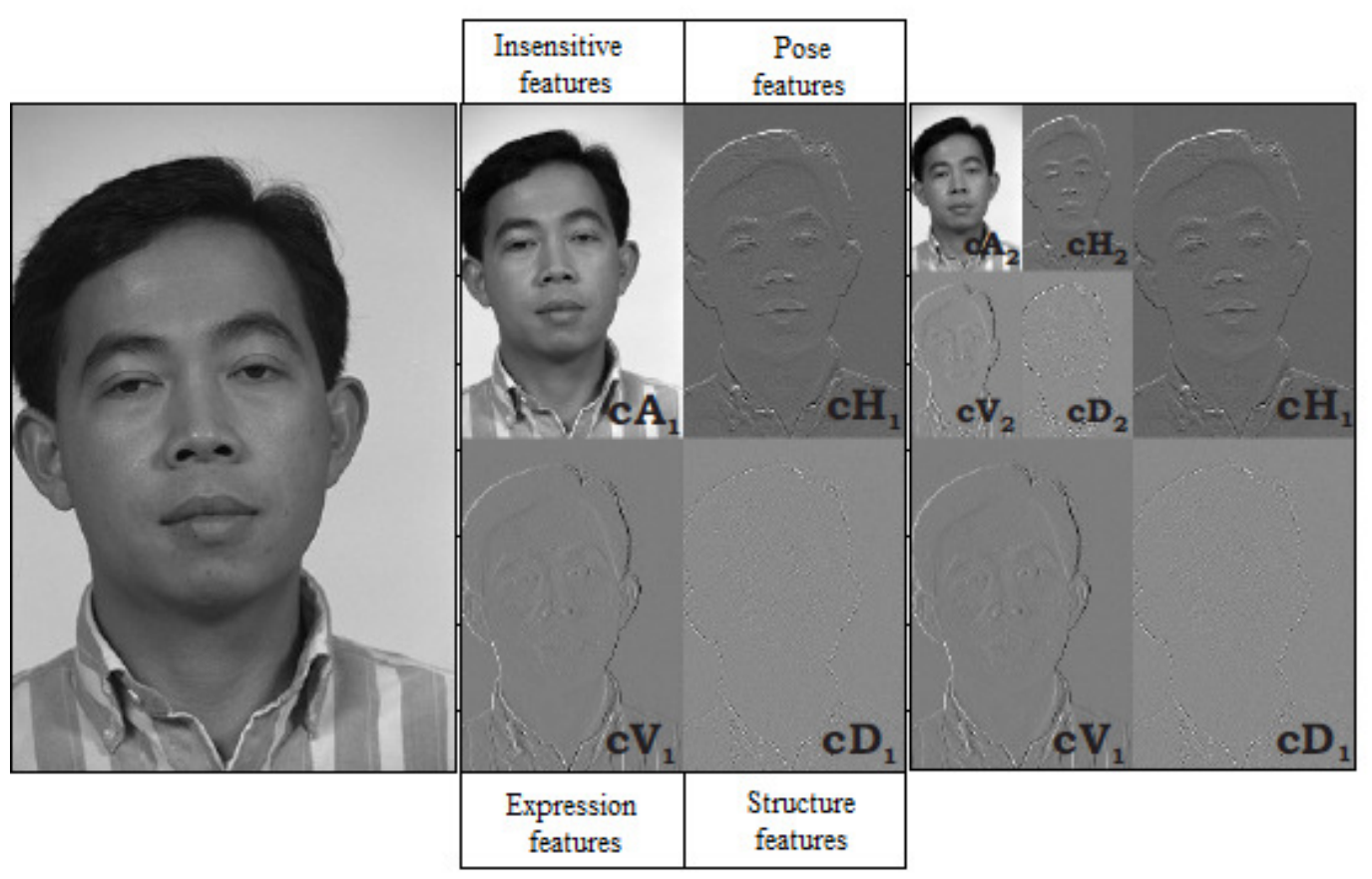

Figure 4: 2D Wavelet decomposition. (a)The Original Image (b) 1-Level Wavelet Decomposition (c) 2-Level Wavelet decomposition. 


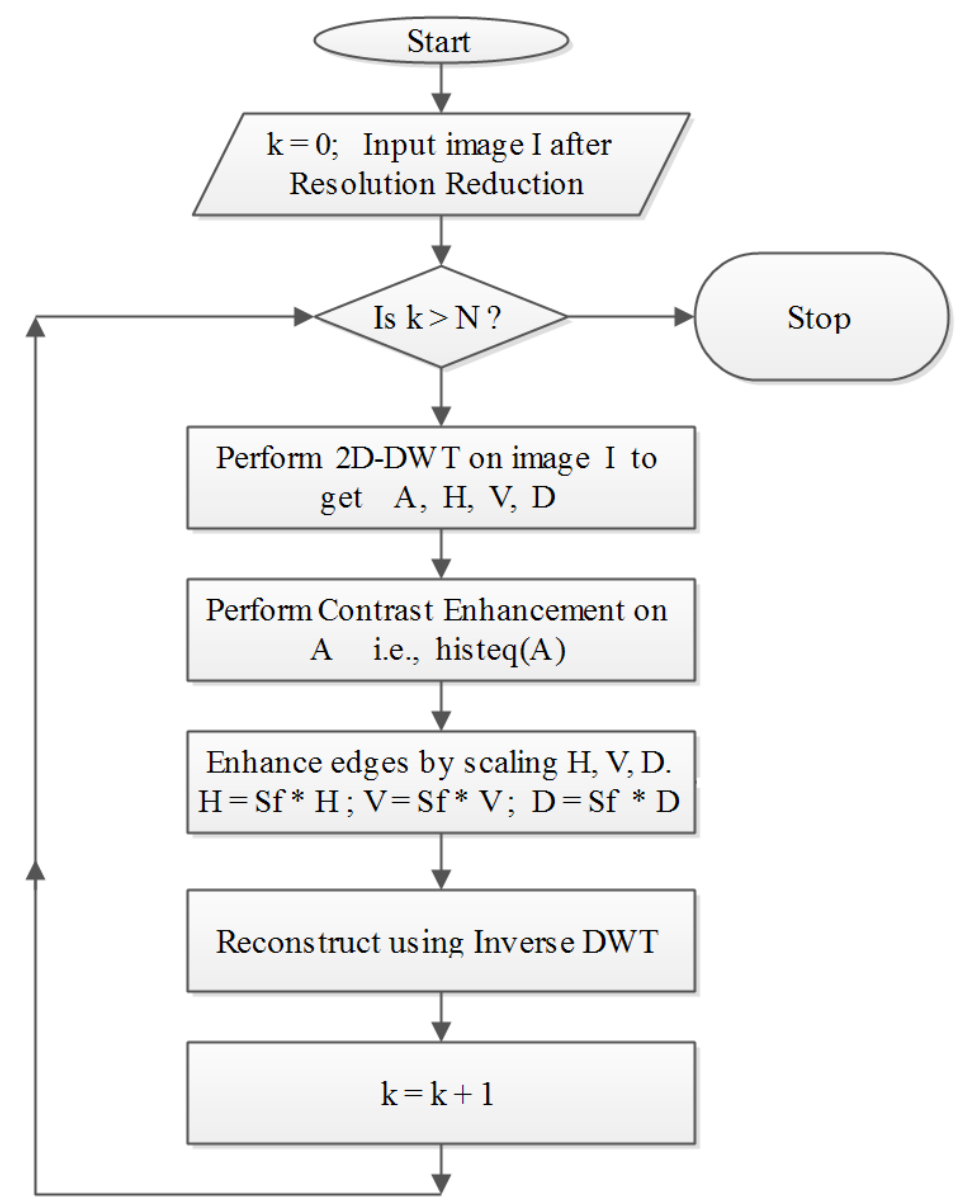

Figure 5: Flowchart of the proposed DWT-MIN with Scale factor Sf.

tical detail, horizontal detail and the diagonal detail coefficients.

3. Adjust the dynamic range of the approximate coefficients to the interval $[0,255]$.

4. Enhance contrast of the approximate coefficients using histogram equalization.

5. Enhance edges of the three detail-coefficient matrices by scaling them with a scaling factor Sf $>1$.

6. Reconstruct the image by applying 2D - IDWT.

7. Adjust the dynamic range of the image to the interval [0, 255].

8. Truncate the ends of its histogram and finally adjust the dynamic range of the image to the interval $[0,255]$. 


\section{Proposed Feature Extraction using DWT}

The Discrete Wavelet Transform (DWT) is a transform which gives both time and frequency information of a signal simultaneously [17, 18, 11]. The basis functions of DWT, wavelets, can be realized using filtering action with rescaling. High pass filtering produces detail information (such as edges) and low pass filtering with scaling produces coarse approximations. The 2-D DWT (Fig. 4) yields four components viz., approximation (cA), horizontal $(\mathrm{cH})$, vertical $(\mathrm{cV})$ and diagonal $(\mathrm{cD})$.

The information in the approximation coefficients band (cA) plays an important role in FR. The edge features are present in the high frequency bands. Unlike other transforms such as Discrete Cosine Transform (DCT) and Discrete Fourier Transform (DFT), DWT not only gives the frequency content of the image but also provides edge localization. We have used DWT for feature extraction to exploit this property.

In this paper, FR using the DWT is based on the facial features extracted from a Haar Wavelet Transform. The Haar wavelet transform is used since it is a powerful technique for multi-resolution decomposition of time series [14].

After all the pre-processing steps (Fig. 3), we apply the 2D-DWT on the edge detected image (Fig. 6). Now the required facial features for recognition are present only in the approximate coefficient band (cA). Only these features are extracted and given to the BPSO block for optimal feature selection.

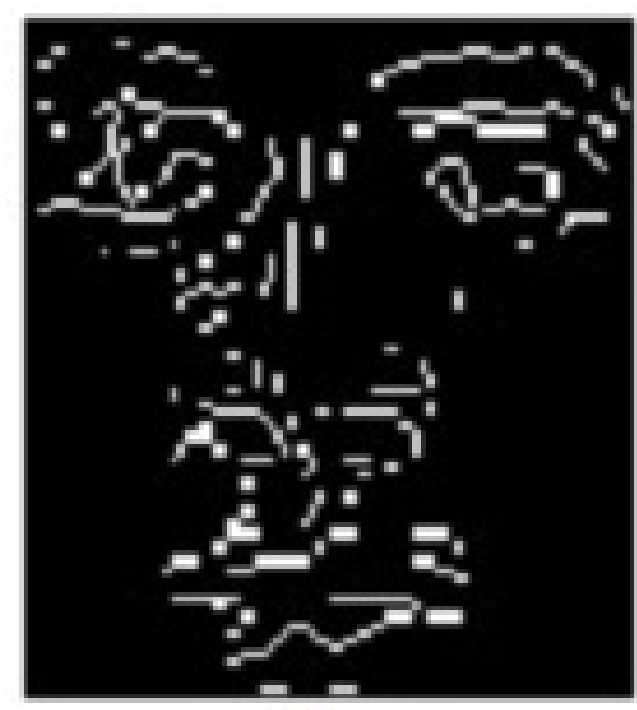

(a)
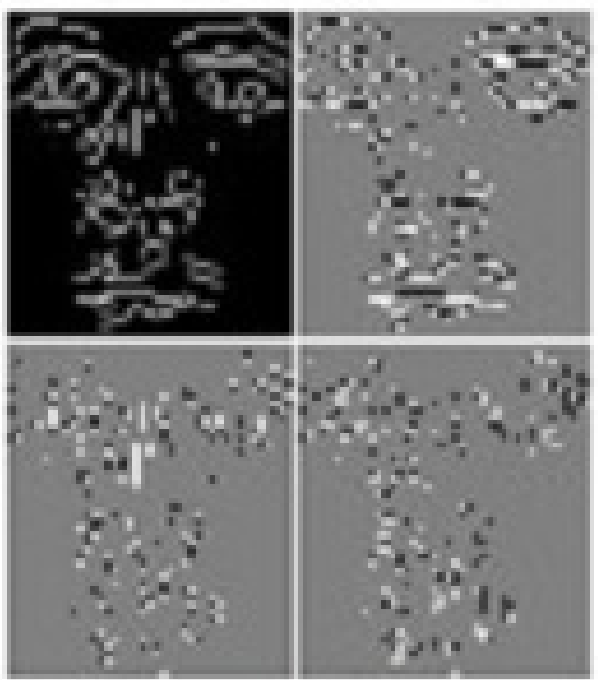

(b)

Figure 6: 2D-DWT as feature extractor. (a) Pre-processed image after edge detection. (b) DWT applied on (a).

To study the effect of DWT-MIN on face recognition rate, we vary the scale 
factor (Sf) used in DWT-MIN and evaluate its effect on various performance metrics such as recognition rate, number of selected features, training and testing times.

\section{Feature Selection}

The feature selection process involves determination of a feature subset which can best represent a given feature set. This is accomplished by the use of binary version of the Particle Swarm Optimization (BPSO).

\subsection{Particle Swarm Optimization (PSO)}

PSO is a computational paradigm which imitates the social behavior of bird flocking. It was originally developed by Kennedy and Eberhart [19]. The performance of each particle is measured by a fitness value which is problem specific. The positions of the particles are updated by evaluating a fitness function to achieve an optimum defined within the search space. The movement of each particle is coordinated by a velocity which has both magnitude and direction. Each particle's position at any instant of time is influenced by its best position and the position of the best particle in a problem space. Particles are updated based on the velocity computed for that particle which draws them towards the global optima. When iterated, the entire swarm converges to the global optima.

The velocity is updated using the following equation:

$$
v_{i d}=v_{i d}+c_{1} * \operatorname{rand}() *\left(p_{i d}-x_{i d}\right)+c_{2} * \operatorname{rand}() *\left(p_{g d}-x_{i d}\right)
$$

where $v_{i d}$ - velocity of the particle, $x_{i d}$ - position of the particle, $p_{i d}$ - pbest of the particle, $p_{g d}$ - gbest achieved by the swarm, $c_{1}$ and $c_{2}$ - cognitive coefficients, $\operatorname{rand}()$ - uniform random number in the range 0 to 1 .

The position updation equation is as follows:

$$
x_{i d}=x_{i d}+v_{i d}
$$

The particles are updated until the termination condition is met. This condition could be either maximum number of iterations or an error goal.

\subsection{Binary Particle Swarm Optimization (BPSO)}

In BPSO [20], each particle's velocity is represented as the probability of bits to change from 0 to 1 . The velocity updation equation is same as that of the 
continuous PSO. The velocity is constrained by using a sigmoid function as given below :

$$
\operatorname{sig}\left(v_{i d}\right)=1 /\left(1+e^{-v_{i d}}\right)
$$

The new position of the particle is obtained as follows:

$$
x_{i d}=\left\{\begin{array}{l}
0, \operatorname{rand}() \geq \operatorname{sig}\left(v_{i d}\right) \\
1, \text { otherwise }
\end{array}\right.
$$

where $\operatorname{rand}()$ is a uniform random number in the range 0 to 1 .

If the particle's position is set to 1 , then the corresponding feature is selected. Otherwise, the feature is discarded. Thus, BPSO plays a major role in minimizing the overall feature set to a smaller feature subset. In this paper, the parameters of BPSO are modified to accomplish the aim of reducing the feature subset at a faster and efficient pace. The parameters of BPSO are swarm size $=30, c_{1}=1.8, c_{2}=2$, inertia is an exponentially decreasing function, and iterations $=100$.

\subsection{Fitness Function}

A fitness function [21, 22] is used so that the class separation is maximized as each particle is evaluated. As the particles are iterated, the quality of the feature subset obtained is ensured to be optimum by checking its ability to maximize the class separation. The fitness value evaluated is the metric that is maximized as the iterations progress. Let $C_{1}, C_{2}, . . C_{N}$ be $\mathrm{N}$ number of classes having $I_{1}, I_{2}, . . \quad I_{L}$ number of images in each respectively. Then the individual class mean is calculated as given below:

$$
M_{k}=\frac{1}{L} \sum_{i=1}^{L} \sum_{j=1}^{S} f_{i k}(j)
$$

where $\mathrm{k}-1,2, \ldots \mathrm{N}, M_{k}$ - individual class mean, L - number of images in each class, S - Size of the feature subset, $f_{i k}(\mathrm{j})$ - selected features of each image.

The class means thus obtained are used to evaluate the grand mean as given below:

$$
M_{o}=\frac{1}{K} \sum_{i=1}^{N} C_{i} M_{i}
$$

where $\mathrm{K}$ is the total number of images used in training.

The proposed fitness function is as follows:

$$
F=\sqrt{\sum_{i=1}^{L}\left(M_{i}-M_{o}\right)^{2}}
$$


Using the PSO-based feature selection algorithm as proposed in [11] leads to a class mean which is an array of selected features. The class separation is dictated by maximizing the fitness value which results in selecting a huge number of features than required for efficient class separation. Since, in classification, individual features are compared, there is a possibility that greater number of features are in common hence leading to a faulty classification. A general mean is used wherein only those features which maximize the class mean are explicitly selected without the knowledge of the individual features of each image. Hence, this helps in significant feature reduction. This plays a key role in the identification of test image during recognition stage. The number of features selected for different databases are presented in Section 7 .

\section{Euclidean classifier}

The feature subset representing each image is the face gallery that is used for similarity measurement in the recognition stage. The test image is also reduced in dimension in order to have the same set of features as the images in the gallery(training images). The P-dimensional Euclidean distance [23] between them is calculated as given below:

$$
F=\sqrt{\sum_{i=1}^{P}\left(f_{i}-t_{i}\right)^{2}}
$$

where $f_{i}$ is the features of the image in the gallery, $t_{i}$ is the selected features of the test image.

\section{Discussion of Proposed FR System and Ex- perimental Results}

Experiments on two benchmark face databases (YaleB and Color FERET) are conducted to evaluate the performance of the proposed FR system (Fig. 1). Recognition rate is used as a performance metric. If there are a total of t test images from all subjects and $c$ images out of these t images can be correctly recognized, then the recognition rate is $\mathrm{c} / \mathrm{t}$.

For example, consider YaleB database having 38 subjects, with 16 images (6 images for training and remaining 10 for testing) per subject. For each test image, let $d_{\min }$ represent the minimum Euclidean distance among the $228(6 \times 38)$ distances calculated. If $d_{\min }$ is found to belong to the proper subject out of 38 subjects, then it represents a correct recognition. This process is 
Table 1: Subsets of Yale B database for Pose 0 (A total of 2432 images)

\begin{tabular}{ccc}
\hline Subsets & Lighting angle (in degrees) & Number of images for 38 subjects \\
\hline 1 & $0-12$ & $7 \times 38=266$ \\
2 & $13-25$ & $12 \times 38=456$ \\
3 & $26-50$ & $12 \times 38=456$ \\
4 & $51-77$ & $14 \times 38=532$ \\
5 & $>77$ & $19 \times 38=722$ \\
\hline
\end{tabular}

repeated for all $380(10 \times 38)$ test images. Thus Recognition rate $=\mathrm{c} / 380$, where $\mathrm{c}$ represents the sum of all correct recognitions.

Two sets of experiments were conducted to evaluate the proposed techniques. The model is tested on Intel Core i5 CPU $2.67 \mathrm{GHz}$ computer with 4.00GB RAM using 64 bit OS and 32-bit MATLAB image processing tool for programming.

\subsection{Experiment 1 : YaleB database}

This database has frontal face images of 38 subjects captured under 64 varying lighting conditions. The original size of each image is $192 \times 168$ pixels. We perform a resolution reduction by 0.5 which reduces the image size to $96 \times$ 84 pixels. There are five subsets [24] according to the light source direction as shown in Table 1.

For our experiment, subset 5 (Fig. 7) is chosen mainly because it has the most number of dark images. It is our goal to show that the proposed FR system works effectively even with these highly illumination variant images. As mentioned in Ref. [24], there are some images that were corrupted during image acquisition; these are not used for our experiments. We are using 16 (out of 19) images per subject of subset 5. Thus a total of 608 images used from subset 5. The block diagram of the proposed FR system for the illumination
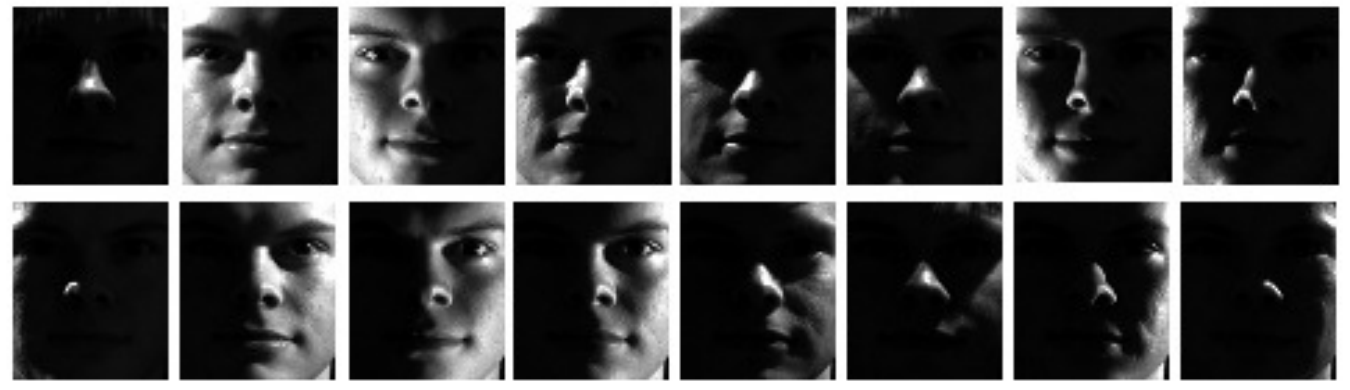

Figure 7: Sample images of Subset 5 of the YaleB database. 
Table 2: Experimental results for YaleB database (Sf- Scale factor, ARR- Average Recognition Rate, ASF - Average number of Selected Features, FS - Feature Selection, ATrT - Average Training Time, ATeT - Average Testing Time).

\begin{tabular}{|c|c|c|c|c|c|c|}
\hline DWT Level & Sf & $\operatorname{ARR}(\%)$ & ASF (before FS) & ASF (after FS) & $\mathrm{ATrT}(\mathrm{s})$ & ATeT per image $\left(\times 10^{-3} \mathrm{~s}\right)$ \\
\hline \multirow{7}{*}{ L1 $(48 \times 42)$} & 1.0 & 97.19 & \multirow{7}{*}{2016} & 1150 & 11.8 & 24.23 \\
\hline & 1.5 & 97.06 & & 1159 & 11.0 & 23.91 \\
\hline & 2.0 & 97.41 & & 1149 & 10.3 & 23.91 \\
\hline & 2.5 & 97.92 & & 1151 & 10.3 & 23.72 \\
\hline & 3.0 & 98.53 & & 1146 & 10.2 & 23.38 \\
\hline & 3.5 & 97.31 & & 1148 & 10.1 & 27.09 \\
\hline & 4.0 & 96.66 & & 1147 & 10.1 & 28.10 \\
\hline \multirow{7}{*}{ L2 $(24 \times 21)$} & 1.0 & 93.38 & \multirow{7}{*}{504} & 328 & 10.0 & 35.45 \\
\hline & 1.5 & 92.77 & & 330 & 9.0 & 34.09 \\
\hline & 2.0 & 76.46 & & 332 & 9.3 & 35.10 \\
\hline & 2.5 & 63.04 & & 335 & 7.5 & 28.64 \\
\hline & 3.0 & 53.12 & & 334 & 7.4 & 29.11 \\
\hline & 3.5 & 45.57 & & 338 & 8.7 & 31.82 \\
\hline & 4.0 & 39.05 & & 337 & 8.3 & 30.61 \\
\hline \multirow{7}{*}{ L3 $(12 \times 11)$} & 1.0 & 75.85 & \multirow{7}{*}{132} & 107 & 7.7 & 38.02 \\
\hline & 1.5 & 45.16 & & 108 & 7.4 & 16.96 \\
\hline & 2.0 & 31.11 & & 109 & 7.0 & 36.28 \\
\hline & 2.5 & 24.17 & & 108 & 7.3 & 40.63 \\
\hline & 3.0 & 21.58 & & 108 & 7.0 & 37.25 \\
\hline & 3.5 & 19.33 & & 109 & 6.6 & 20.85 \\
\hline & 4.0 & 16.86 & & 109 & 6.6 & 35.38 \\
\hline
\end{tabular}

variant images is shown in Fig. 1.

Table 2 and Fig. 8 show the experimental results as a function of scale factor, Sf, used in the proposed DWT-MIN, and wavelet decomposition levels, L1 to L3. The 16 images of each subject are used for training and testing

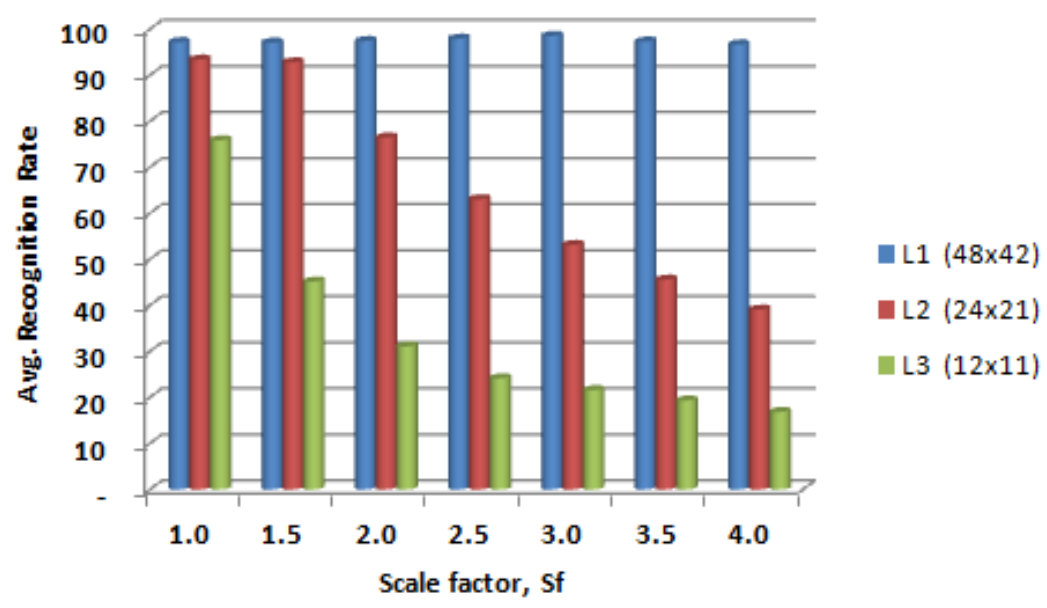

Figure 8: Recognition rate vs Scale factor for YaleB. 
Table 3: Results for varying Train:Test ratios for YaleB database (Subset 5), DWT level L1, Scale factor $\mathrm{Sf}=3.0$

\begin{tabular}{ccccc}
\hline Train: Test & ARR $(\%)$ & Peak RR $(\%)$ & ATrT $(\mathrm{s})$ & ATeT per image $\left(\times 10^{-3} \mathrm{~s}\right)$ \\
\hline $1: 15$ & 85.70 & 91.75 & 8.4 & 25.61 \\
$2: 14$ & 94.85 & 96.80 & 9.6 & 24.92 \\
$3: 13$ & 98.53 & 98.79 & 10.2 & 23.38 \\
$4: 12$ & 98.61 & 99.56 & 12.6 & 23.11 \\
$5: 11$ & 99.21 & 100 & 15.4 & 22.73 \\
$6: 10$ & 99.50 & 100 & 18.9 & 21.84 \\
$7: 9$ & 99.53 & 100 & 22.8 & 20.66 \\
$8: 8$ & 99.84 & 100 & 26.4 & 20.02 \\
$9: 7$ & 99.70 & 100 & 31.4 & 19.23 \\
$10: 6$ & 99.74 & 100 & 34.7 & 17.33 \\
$11: 5$ & 99.89 & 100 & 37.2 & 15.08 \\
$12: 4$ & 100 & 100 & 39.9 & 12.43 \\
$13: 3$ & 99.91 & 100 & 41.7 & 9.55 \\
$14: 2$ & 100 & 100 & 44.8 & 6.64 \\
$15: 1$ & 100 & 100 & 47.7 & 4.96 \\
\hline
\end{tabular}

randomly in the ratio $3: 13$. These results show that decomposition level L1 and scale factor $\mathrm{Sf}=3.0$, give the best Average Recognition Rate (ARR) of 98.53\%. The corresponding selected features after BPSO is found to be 1146 features.

Table 3 shows the results for different ratios of training and testing images. The wavelet decomposition level and scale factor are kept constant at L1 and 3.0 respectively.

\subsection{Experiment 2 : Color FERET database}

Color FERET Database contains face images of 994 subjects. The original size of each image is $768 \times 512$ pixels [25]. We perform a resolution reduction by 0.5 which reduces the image size to $384 \times 256$ pixels. There are a total of 13 pose variations as shown in Table 4 and Fig. 9 .

The block diagram of the proposed FR system is shown in Fig. 1 which applies to both YaleB and Color FERET. In this experiment, two subsets are created. Subset 1 includes two images ( $\mathrm{fa}$ and $\mathrm{fb}$ ) of 35 subjects. Subset 2 includes four images ( $\mathrm{fa}, \mathrm{fb}, \mathrm{rb}$, and $\mathrm{rc}$ ) of 35 subjects. Subset 2 is used to study the performance for pose variant images. The sample images of fa, fb, rb and rc are shown in Fig. 10. 
Table 4: Pose variations in Color FERET database

\begin{tabular}{ll}
\hline Pose & Description \\
\hline fa & regular frontal image \\
$\mathrm{fb}$ & alternative frontal image, taken shortly after the corresponding fa image \\
$\mathrm{pl}$ & profile left \\
$\mathrm{hl}$ & half left - head turned about 67.5 degrees left \\
$\mathrm{ql}$ & quarter left - head turned about 22.5 degrees left \\
$\mathrm{pr}$ & profile right \\
$\mathrm{hr}$ & half right - head turned about 67.5 degrees right \\
$\mathrm{qr}$ & quarter right - head turned about 22.5 degrees right \\
$\mathrm{ra}$ & random image - head turned about 45 degrees left \\
$\mathrm{rb}$ & random image - head turned about 15 degrees left \\
$\mathrm{rc}$ & random image - head turned about 15 degrees right \\
$\mathrm{rd}$ & random image - head turned about 45 degrees right \\
re & random image - head turned about 75 degrees right \\
\hline
\end{tabular}

\subsubsection{Experiment 2a : Subset 1 (fa, fb)}

Table 5 and Fig. 11 show the recognition results as a function of scale factor Sf used in illumination normalization, and wavelet decomposition levels L1 to

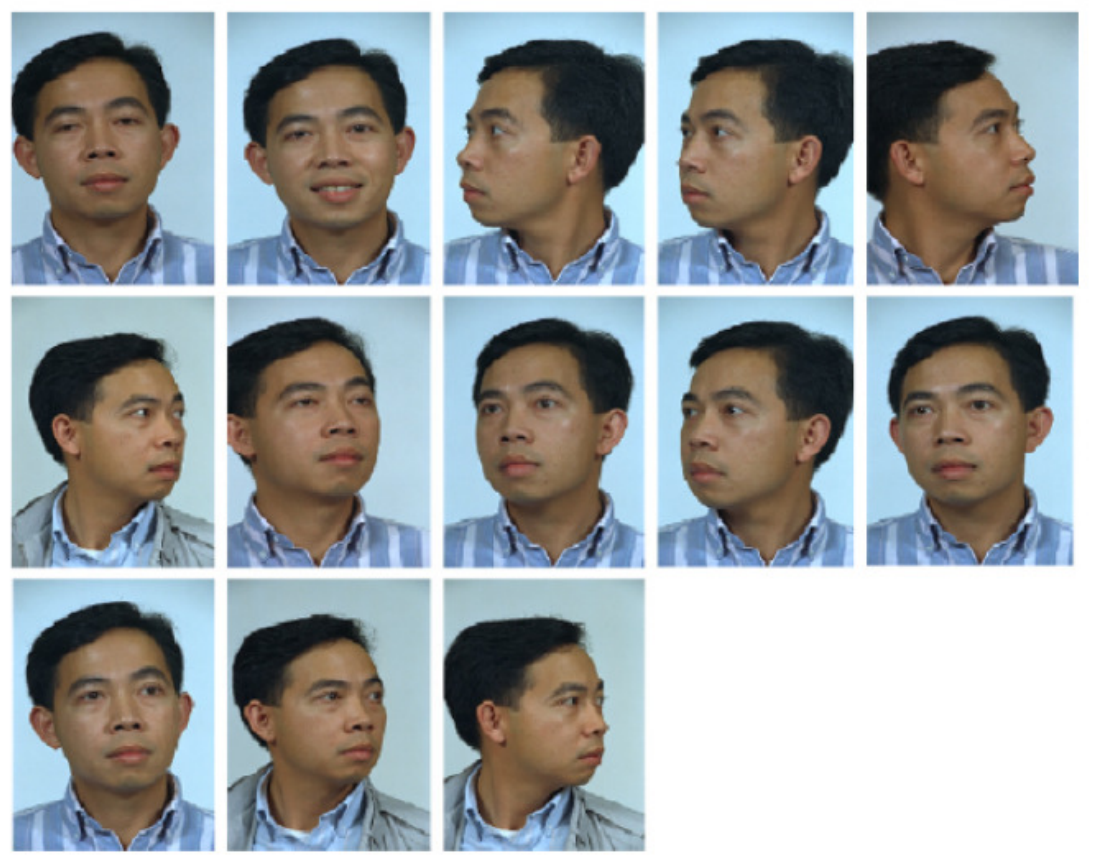

Figure 9: Sample images of a person from FERET database under 13 different poses. 
L6. For each subject, there is one image in fa and one image in fb and all images are of frontal pose. Out of these 2, one image is chosen randomly for training and the other one for testing (thus, training : testing ratio is 1:1).

\subsubsection{Experiment $2 \mathrm{~b}$ : Subset $2(\mathrm{fa}, \mathrm{fb}, \mathrm{rb}, \mathrm{rc})$}

Table 6 and Fig. 12 show the recognition results as a variation of scale factor Sf used in illumination normalization, and wavelet decomposition levels L1 to L6. For each person, there is one image each in $\mathrm{fa}, \mathrm{fb}, \mathrm{rb}, \mathrm{rc}$. Out of these 4, two images are chosen randomly for training and the remaining two for testing (thus, training : testing ratio is 2:2).

Table 7 shows the results for different ratios of training to testing images. The wavelet decomposition level and scale factor are kept constant at L6 and 3.0 respectively.

\section{Comparison with other FR systems}

For YaleB Database, Table 8 and Fig. 13 shows the comparison of the recognition rate values with $50 \%$ resolution, wavelet decomposition level L1 and scale factor $\mathrm{Sf}=3.0$. These results are compared with those from Complex dual tree DWT (C-DT-DWT) 9], Local Binary Patterns (LBP), Curvelet and Contourlet approaches, Dual Tree Complex Wavelet Transform (DTCWT), Self-Quotient Image (SQI), Logarithmic Total variation (LTV), Logarithmic Wavelet Transform (LWT), Logarithmic Non-subsampled Contourlet Transform (LNSCT) [26] and the Tan and Triggs [27] technique. The results show a significant improvement as compared to other methods.

For Color FERET Database (fa,fb), Table 8 and Fig. 14 shows the comparison of the ARR values with 50\% resolution, wavelet decomposition level L6

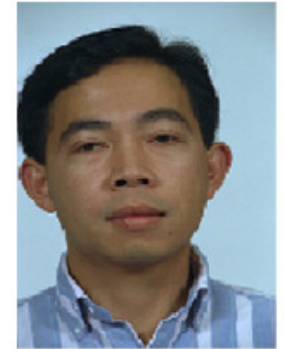

fa

$\left(0^{\circ}\right)$

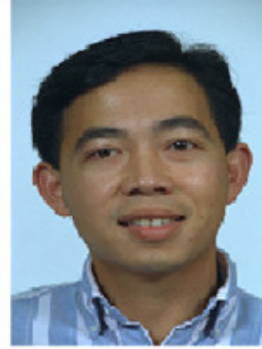

$\mathrm{fb}$

$\left(0^{\circ}\right)$

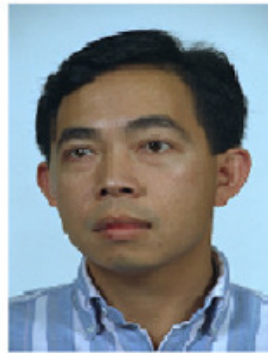

$\mathrm{rb}$

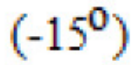

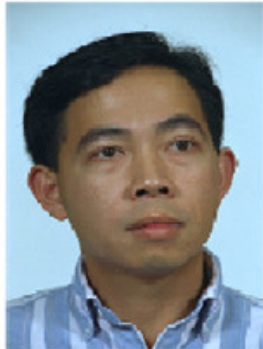

IC

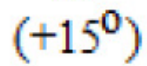

Figure 10: FERET images for our experimentation. 


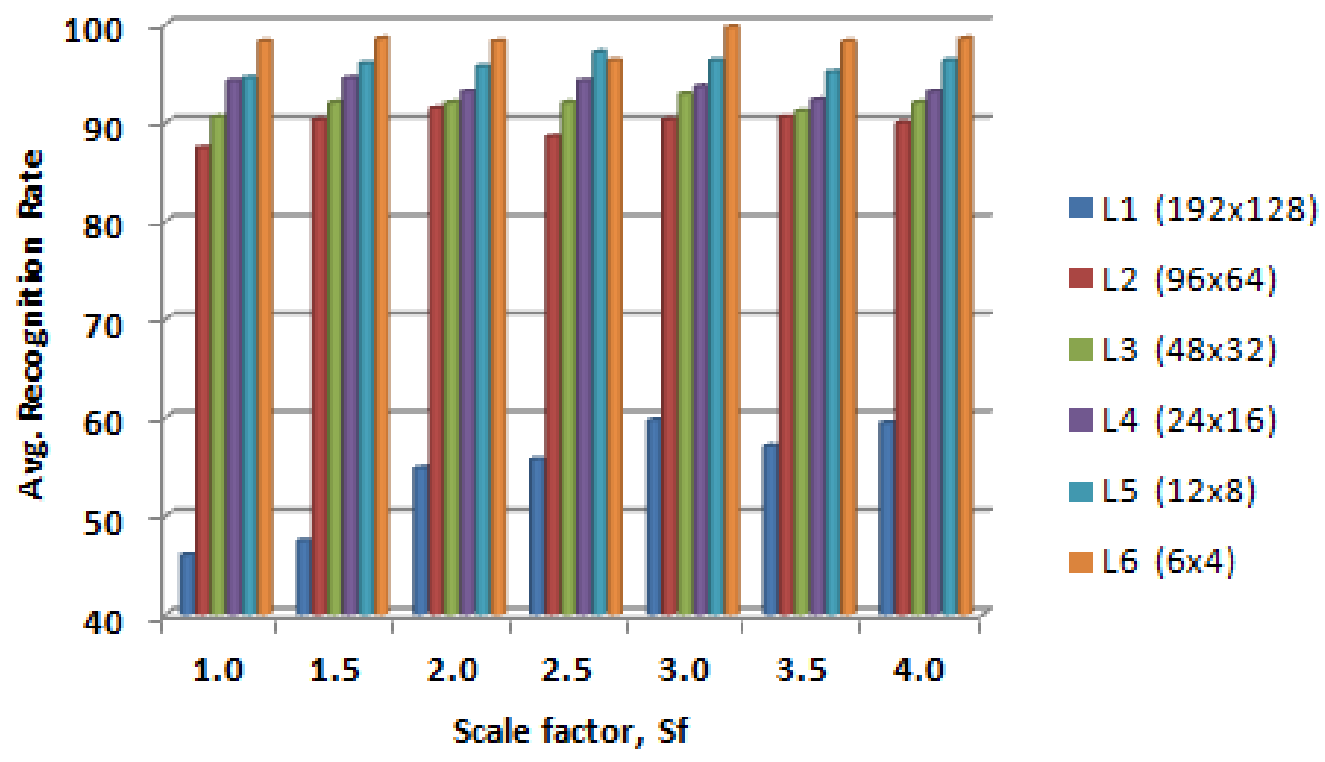

Figure 11: Recognition rate vs Scale factor for Color FERET fafb.

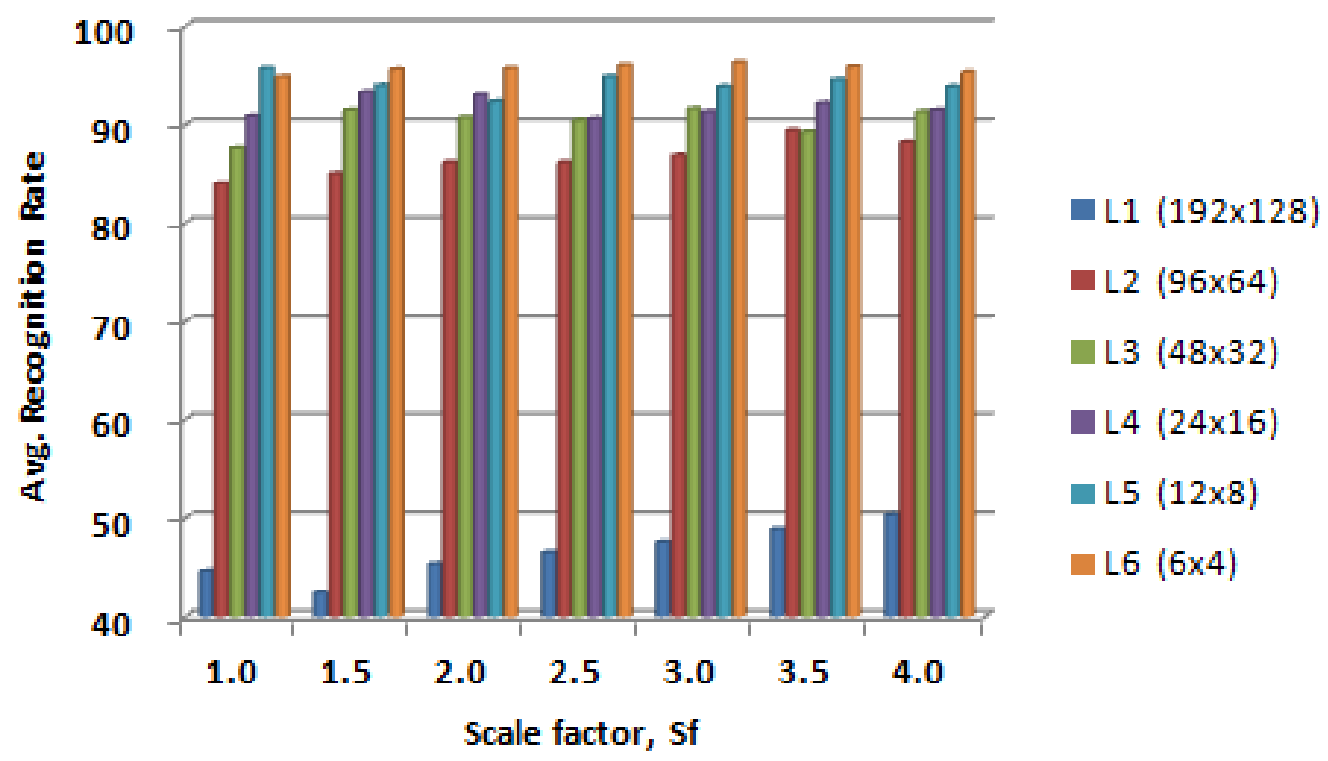

Figure 12: Recognition rate vs Scale factor for Color FERET fafbrbrc. 
Table 5: Results for Color FERET (fa, fb) database.

\begin{tabular}{|c|c|c|c|c|c|c|}
\hline DWT Level & $\mathrm{Sf}$ & $\operatorname{ARR}(\%)$ & ASF (before FS) & ASF (after FS) & $\mathrm{ATrT}(\mathrm{s})$ & ATeT per image $\left(\times 10^{-2} \mathrm{~s}\right)$ \\
\hline \multirow{7}{*}{ L1 $(192 \times 128)$} & 1.0 & 46.00 & \multirow{7}{*}{24576} & 12831 & 65.8 & 15.37 \\
\hline & 1.5 & 47.43 & & 12838 & 65.8 & 16.54 \\
\hline & 2.0 & 54.86 & & 12837 & 69.3 & 17.54 \\
\hline & 2.5 & 55.71 & & 12862 & 75.2 & 19.46 \\
\hline & 3.0 & 59.71 & & 12861 & 71.1 & 17.77 \\
\hline & 3.5 & 57.14 & & 12848 & 71.8 & 17.97 \\
\hline & 4.0 & 59.43 & & 12848 & 73.2 & 18.46 \\
\hline \multirow{7}{*}{ L2 $(96 \times 64)$} & 1.0 & 87.43 & \multirow{7}{*}{6144} & 3367 & 29.6 & 22.29 \\
\hline & 1.5 & 90.29 & & 3368 & 29.3 & 21.63 \\
\hline & 2.0 & 91.43 & & 3369 & 29.7 & 21.71 \\
\hline & 2.5 & 88.57 & & 3365 & 30.4 & 22.51 \\
\hline & 3.0 & 90.29 & & 3374 & 32.3 & 21.77 \\
\hline & 3.5 & 90.57 & & 3379 & 30.3 & 21.26 \\
\hline & 4.0 & 90.00 & & 3373 & 29.5 & 20.26 \\
\hline \multirow{7}{*}{ L3 $(48 \times 32)$} & 1.0 & 90.57 & \multirow{7}{*}{1536} & 920 & 14.4 & 21.89 \\
\hline & 1.5 & 92.00 & & 922 & 15.4 & 23.46 \\
\hline & 2.0 & 92.00 & & 923 & 15.5 & 22.89 \\
\hline & 2.5 & 92.00 & & 922 & 14.9 & 22.20 \\
\hline & 3.0 & 92.86 & & 924 & 15.6 & 22.77 \\
\hline & 3.5 & 91.14 & & 922 & 15.4 & 22.60 \\
\hline & 4.0 & 92.00 & & 924 & 14.8 & 22.60 \\
\hline \multirow{7}{*}{ L4 $(24 \times 16)$} & 1.0 & 94.29 & \multirow{7}{*}{384} & 266 & 14.0 & 26.46 \\
\hline & 1.5 & 94.57 & & 268 & 14.0 & 27.80 \\
\hline & 2.0 & 93.14 & & 268 & 14.6 & 27.29 \\
\hline & 2.5 & 94.29 & & 269 & 15.3 & 27.46 \\
\hline & 3.0 & 93.71 & & 269 & 15.1 & 27.69 \\
\hline & 3.5 & 92.29 & & 267 & 15.0 & 27.54 \\
\hline & 4.0 & 93.14 & & 272 & 15.2 & 27.57 \\
\hline \multirow{7}{*}{ L5 $(12 \times 8)$} & 1.0 & 94.57 & \multirow{7}{*}{96} & 83 & 14.9 & 30.66 \\
\hline & 1.5 & 96.00 & & 83 & 15.0 & 31.11 \\
\hline & 2.0 & 95.71 & & 84 & 15.5 & 30.69 \\
\hline & 2.5 & 97.14 & & 84 & 15.1 & 30.31 \\
\hline & 3.0 & 96.29 & & 83 & 15.2 & 30.54 \\
\hline & 3.5 & 95.14 & & 81 & 15.2 & 30.63 \\
\hline & 4.0 & 96.29 & & 83 & 15.6 & 29.91 \\
\hline \multirow{7}{*}{ L6 $(6 \times 4)$} & 1.0 & 98.29 & \multirow{7}{*}{24} & 21 & 15.7 & 33.66 \\
\hline & 1.5 & 98.57 & & 21 & 16.0 & 34.46 \\
\hline & 2.0 & 98.29 & & 21 & 15.8 & 34.49 \\
\hline & 2.5 & 96.29 & & 21 & 16.0 & 35.23 \\
\hline & 3.0 & 99.71 & & 21 & 16.6 & 40.49 \\
\hline & 3.5 & 98.29 & & 21 & 15.7 & 35.51 \\
\hline & 4.0 & 98.57 & & 21 & 15.5 & 34.83 \\
\hline
\end{tabular}

and scale factor $\mathrm{Sf}=3.0$. These results are compared with those from C-DTDWT [9], Semi Random Subspace (Semi-RS) [28] and Fisher-LDA (FLDA) [29]. The results show a substantial improvement in ARR of the proposed method as compared to other methods. 
Table 6: Results for Color FERET (fa, fb, rb, rc) database.

\begin{tabular}{|c|c|c|c|c|c|c|}
\hline DWT Level & $\mathrm{Sf}$ & $\operatorname{ARR}(\%)$ & ASF (before FS) & ASF (after FS) & $\mathrm{ATrT}(\mathrm{s})$ & ATeT per image $\left(\times 10^{-2} \mathrm{~s}\right)$ \\
\hline \multirow{7}{*}{ L1 $(192 \times 128)$} & 1.0 & 44.57 & \multirow{7}{*}{24576} & 12863 & 98.5 & 18.73 \\
\hline & 1.5 & 42.43 & & 12861 & 96.3 & 18.90 \\
\hline & 2.0 & 45.29 & & 12892 & 94.8 & 18.16 \\
\hline & 2.5 & 46.43 & & 12860 & 95.2 & 19.11 \\
\hline & 3.0 & 47.57 & & 12881 & 97.9 & 19.54 \\
\hline & 3.5 & 48.86 & & 12869 & 96.6 & 19.24 \\
\hline & 4.0 & 50.29 & & 12876 & 96.5 & 18.09 \\
\hline \multirow{7}{*}{ L2 $(96 \times 64)$} & 1.0 & 84.00 & \multirow{7}{*}{6144} & 3370 & 43.0 & 20.46 \\
\hline & 1.5 & 85.00 & & 3370 & 42.4 & 19.93 \\
\hline & 2.0 & 86.14 & & 3381 & 42.4 & 19.70 \\
\hline & 2.5 & 86.14 & & 3370 & 42.5 & 20.03 \\
\hline & 3.0 & 86.86 & & 3382 & 43.0 & 20.36 \\
\hline & 3.5 & 89.43 & & 3369 & 42.2 & 20.01 \\
\hline & 4.0 & 88.14 & & 3373 & 42.6 & 19.90 \\
\hline \multirow{7}{*}{ L3 $(48 \times 32)$} & 1.0 & 87.57 & \multirow{7}{*}{1536} & 921 & 24.3 & 22.64 \\
\hline & 1.5 & 91.43 & & 924 & 24.7 & 23.49 \\
\hline & 2.0 & 90.71 & & 920 & 24.7 & 23.06 \\
\hline & 2.5 & 90.43 & & 920 & 25.8 & 23.29 \\
\hline & 3.0 & 91.57 & & 922 & 24.4 & 23.56 \\
\hline & 3.5 & 89.29 & & 926 & 25.3 & 23.09 \\
\hline & 4.0 & 91.29 & & 922 & 24.7 & 23.36 \\
\hline \multirow{7}{*}{ L4 $(24 \times 16)$} & 1.0 & 90.86 & \multirow{7}{*}{384} & 270 & 23.7 & 26.56 \\
\hline & 1.5 & 93.29 & & 269 & 23.6 & 26.20 \\
\hline & 2.0 & 93.00 & & 271 & 23.3 & 26.34 \\
\hline & 2.5 & 90.57 & & 267 & 23.2 & 26.33 \\
\hline & 3.0 & 91.29 & & 272 & 23.0 & 26.51 \\
\hline & 3.5 & 92.14 & & 270 & 22.9 & 26.14 \\
\hline & 4.0 & 91.43 & & 269 & 23.8 & 26.34 \\
\hline \multirow{7}{*}{ L5 $(12 \times 8)$} & 1.0 & 95.71 & \multirow{7}{*}{96} & 82 & 24.4 & 29.71 \\
\hline & 1.5 & 94.00 & & 82 & 24.7 & 29.69 \\
\hline & 2.0 & 92.29 & & 82 & 24.5 & 29.44 \\
\hline & 2.5 & 94.86 & & 82 & 24.9 & 30.03 \\
\hline & 3.0 & 93.86 & & 82 & 24.7 & 29.60 \\
\hline & 3.5 & 94.57 & & 83 & 25.0 & 29.99 \\
\hline & 4.0 & 93.86 & & 82 & 25.9 & 31.73 \\
\hline \multirow{7}{*}{ L6 $(6 \times 4)$} & 1.0 & 94.86 & \multirow{7}{*}{24} & 21 & 30.4 & 37.03 \\
\hline & 1.5 & 95.57 & & 21 & 29.9 & 35.83 \\
\hline & 2.0 & 95.71 & & 21 & 26.4 & 33.86 \\
\hline & 2.5 & 96.00 & & 21 & 29.3 & 34.81 \\
\hline & 3.0 & 96.29 & & 21 & 27.3 & 32.59 \\
\hline & 3.5 & 95.86 & & 21 & 25.8 & 31.90 \\
\hline & 4.0 & 95.29 & & 21 & 31.7 & 38.57 \\
\hline
\end{tabular}

Table 7: Results for varying Train:Test ratios for Color FERET database (fa, fb, $\mathrm{rb}, \mathrm{rc})$, DWT level L6, Scale factor $\mathrm{Sf}=3.0$

\begin{tabular}{ccccc}
\hline Train: Test & ARR $(\%)$ & Peak RR $(\%)$ & ATrT $(\mathrm{s})$ & ATeT per image $\left(\times 10^{-2} \mathrm{~s}\right)$ \\
\hline $1: 3$ & 85.43 & 90.48 & 19.8 & 62.17 \\
$2: 2$ & 96.29 & 100 & 27.3 & 32.59 \\
$3: 1$ & 98.00 & 100 & 51.3 & 22.06 \\
\hline
\end{tabular}


Table 8: Comparison of RR with other FR systems

\begin{tabular}{ll|ll}
\hline \multicolumn{2}{c|}{ YaleB (Subset 5) } & \multicolumn{2}{c}{ Color FERET (fa, fb) } \\
\hline Method & ARR (\%) & Method & ARR (\%) \\
\hline Curvelet [26] & 29.73 & C-DT-DWT [9] & 73.66 \\
LBP [26] & 31.75 & Semi-RS [28] & 88.80 \\
DT-CWT [26] & 32.34 & FLDA [29] & 90.50 \\
Contourlet [26] & 35.87 & Proposed Method & $\mathbf{9 9 . 7 1}$ \\
SQI [26] & 81.61 & & \\
C-DT-DWT [9] & 88.55 & & \\
LWT [26] & 90.59 & & \\
LTV [26] & 90.97 & & \\
Tan and Triggs [27] & 97.20 & & \\
LNSCT [26] & 98.20 & & \\
Proposed Method & $\mathbf{9 8 . 5 3}$ & & \\
\hline
\end{tabular}

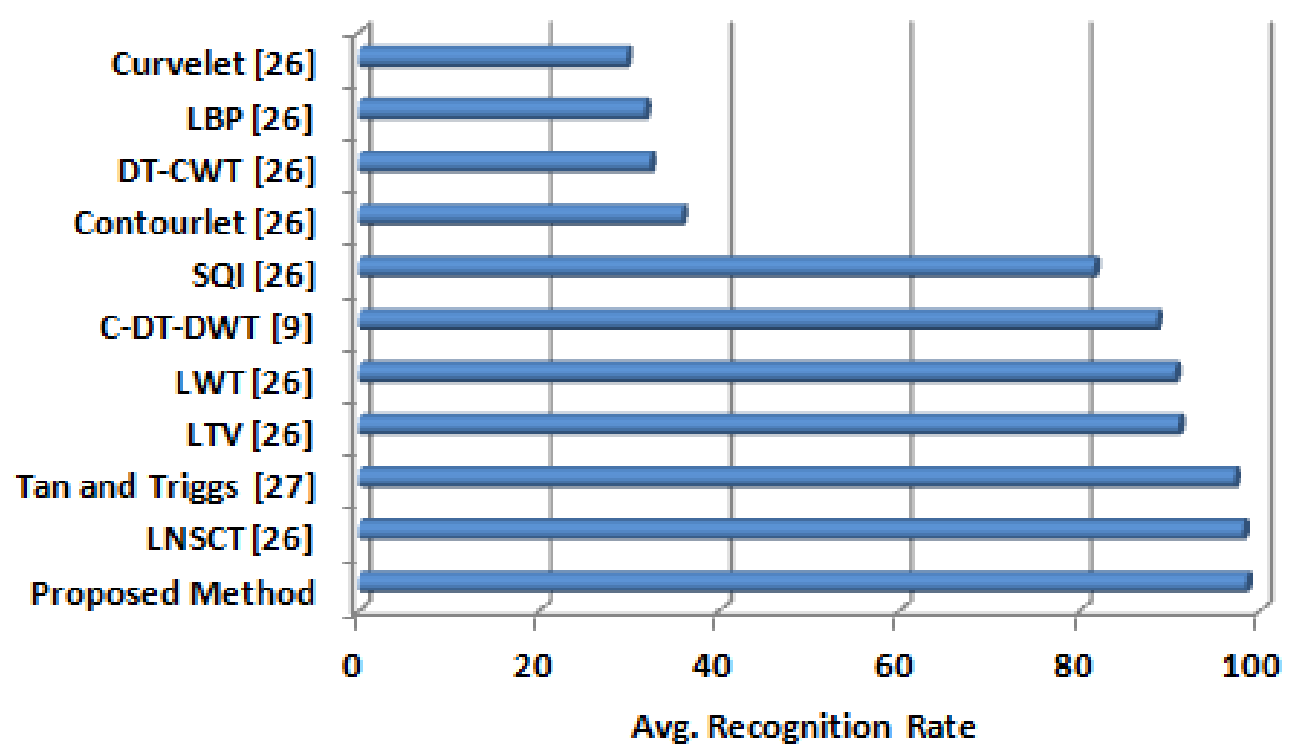

Figure 13: Comparison results for YaleB.

\section{Conclusions}

A novel approach for a flexible FR system is proposed which uses DWT for Multilevel Illumination Normalization (DWT-MIN) as well as for feature extraction. It is found that DWT-MIN with scale factor $\mathrm{Sf}=3.0$ gives the best Recognition Rate at each DWT decomposition level. Feature selection based on BPSO significantly reduces the number of features selected, thus reducing the computational cost. DWT has played a key role in efficient feature 


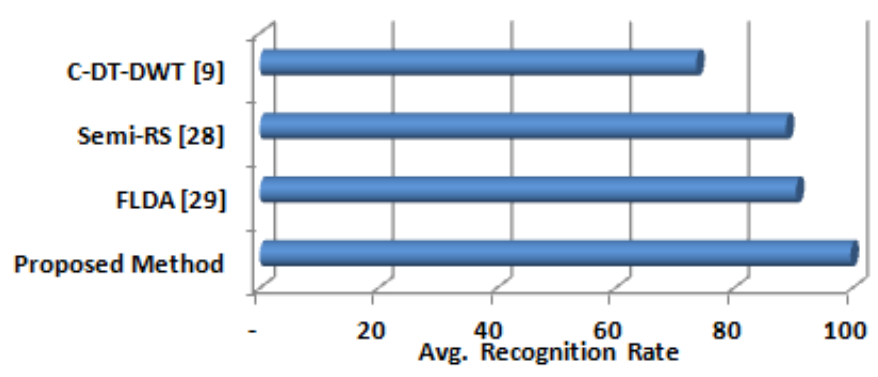

Figure 14: Comparison results for Color FERET fafb.

extraction which is the main contributor for the high recognition rates being obtained. A successful attempt has been made to equally handle all image variations (illumination, facial expressions, pose and surroundings). The proposed method exhibits extremely good performance under frontal poses with variations in facial expressions and facial details (FERETfafb). The experimental results indicate that the proposed method has performed well under severe illumination variations with top recognition rate having reached $100 \%$ for subset 5 of Yale B considering only pose 0 . Thus, the proposed method is efficient in practical situations where the images may be taken in uncontrolled and unknown surroundings. It is also successful in tackling the most challenging task of pose variance in $\mathrm{FR}$, with average recognition rate of $96.29 \%$ for FERET database (considering 3 poses) for DWT level 6. Thus, the proposed method has proven to be a promising technique under arbitrary variations in illumination, poses and backgrounds with slight occlusions too.

\section{References}

[1] W. Zhao, R. Chellappa, P.J. Phillips, A. Rosenfeld, Face recognition: A literature survey, ACM Computing Surveys 35 (2003) 399-458.

[2] A.M. Patil, S.R. Kolhe, P.M. Patil, 2D Face recognition techniques: A survey, International Journal of Machine Intelligence 2 (2010) 74-83.

[3] Xuan Zou, J. Kittler, K. Messer, Illumination invariant face recognition: A survey, First IEEE International Conference on Biometrics: Theory, Applications, and Systems (2007).

[4] Shiguang Shan, Wen Gao, Bo Cao, Debin Zhao, Illumination normalization for robust face recognition against varying lighting conditions, Analysis and Modelling of Faces and Gestures, Proceedings of IEEE International Workshop (2003). 
[5] Shan Du, Rabab Ward, Wavelet-based illumination normalization for face recognition, Proceedings of IEEE International Conference on Image Processing (ICIP) (2005).

[6] Lior Shamir, Pose and Illumination Variance with Compound image transforms, Advances in Face image Analysis: Techniques and Technologies, IGI Global (2011) 301-315.

[7] V.P. Vishwakarma, S. Pandey, M.N. Gupta, An Illumination Invariant Accurate Face Recogntion with Down Scaling of DCT Coefficients, Journal of Computing and Information Technology (2010) 53-67.

[8] Vitomir Struc, Nikola Pavesic, Photometric normalization techniques for illumination invariance, Advances in Face image Analysis: Techniques and Technologies, IGI Global (2011) 279-300.

[9] A.A. Bhurane, S.N. Talbar, P.N. Gophane, Comparative Analysis of Conventional, Real and Complex Wavelet Transforms for Face Recognition, International Journal of Computer Applications 39 (2012) 6-12.

[10] C.M. Mohan, V.V. Kumar, V.V. Krishna, Novel Method of Adult Age Classification using Linear Wavelet Transforms, International Journal of Computer Science and Network Security 10 (2010).

[11] Rabab M. Ramadan, Rehab F. Abdel Kader, Face Recognition Using Particle Swarm Optimization-Based Selected Features, International Journal of Signal Processing, Image Processing and Pattern Recognition 2 (2009) 5165.

[12] Rafael C. Gonzalez, Richard E. Woods, Digital Image Processing, Third Edition, Prentice Hall, 2008; 133-135, 242-275, 485-488.

[13] John Canny, A Computational Approach to Edge Detection, IEEE Transactions on pattern analysis and machine intelligence PAMI-8 (1986).

[14] 〈http://www . mathworks . com>.

[15] Vitomir Struc, Nikola Pavesic, Gabor based partial least squares discrimination features for face recognition, Informatica 20 (2009) 115-138.

[16] M.A. Anjum, M.Y. Javed, A. Basit, A New Approach to Face Recognition using Dual Dimension Reduction, International Journal of Signal Processing 2 (2005) 1-6.

[17] I. Daubechies, The wavelet transform, time-frequency localization and signal analysis, IEEE Trans. on Information Theory 36 (1990) 961-1005. 
[18] A.S. Samra, S.E. Gad Allah, R.M. Ibrahim, Face Recognition Using Wavelet Transform, Fast Fourier Transform and Discrete Cosine Transform, Proceedings of 46th IEEE International Midwest Symposium on Circuits and Systems (MWSCAS'03) 1 (2003) 272-275.

[19] J. Kennedy, R.C. Eberhart, Particle swarm optimization, Proceedings of the International Conference on Neural Networks, Piscataway, NJ (1995).

[20] J. Kennedy, R.C. Eberhart, A discrete binary version of the particle swarm algorithm, Proceedings of the World Multiconference on Systemics, Cybernetics and Informatics, Piscataway, NJ (1997).

[21] Xiaohui Hu, Yuhui Shi, R. Eberhart, Recent advances in particle swarm, Proceedings of IEEE Congress on Evolutionary Computation (2004).

[22] C. Liu, H. Wechsler, Evolutionary Pursuit and Its Application to Face Recognition, IEEE Transactions on Pattern Analysis and Machine Intelligence 22 (2000) 570-582.

[23] Subash Chelliah, A Study of Euclidean classifier, Remote Sensing Division, Birla Institute of Scientific Research, Jaipur, India, 1989.

[24] Yale Univ. Face Database: <http://cvc.yale.edu/projects/ yalefaces/yalefaces.html>.

[25] Color FERET database: 〈http://face.nist.gov/colorferet>.

[26] Xiaohua Xie, Jianhuang Lai, Wei-Shi Zheng, Extraction of illumination invariant facial features from a single image using non sub-sampled contourlet transform, Pattern Recognition 43 (2010) 4177-4189.

[27] Raghuraman Gopalan, David Jacobs, Comparing and combining lighting insensitive approaches for face recognition, Computer Vision and Image Understanding 114 (2010) 135-145.

[28] Yulian Zhu, Jun Liu, Songcan Chen, Semi-random subspace method for face recognition, Image and Vision Computing 27 (2009) 1358-1370.

[29] Quan-xue Gao, Lei Zhang, David Zhang, Face recognition using FLDA with single training image per person, Applied Mathematics and Computation 205 (2008) 726-734. 